\title{
Polyethylene glycol versus dual sugar assay for gastrointestinal permeability analysis: is it time to choose?
}

This article was published in the following Dove Press journal:

Clinical and Experimental Gastroenterology

18 July 2012

Number of times this article has been viewed

\author{
Kim van Wijck ${ }^{1,2}$ \\ Babs AFM Bessems ${ }^{2}$ \\ Hans $M H$ van Eijk ${ }^{2}$ \\ Wim A Buurman ${ }^{2}$ \\ Cornelis HC Dejong ${ }^{1,2}$ \\ Kaatje Lenaerts ${ }^{1,2}$ \\ 'Top Institute Food and Nutrition, \\ Wageningen, The Netherlands; \\ ${ }^{2}$ Department of Surgery, NUTRIM \\ School for Nutrition, Toxicology and \\ Metabolism, Maastricht University \\ Medical Center, Maastricht, \\ The Netherlands
}

Background: Increased intestinal permeability is an important measure of disease activity and prognosis. Currently, many permeability tests are available and no consensus has been reached as to which test is most suitable. The aim of this study was to compare urinary probe excretion and accuracy of a polyethylene glycol (PEG) assay and dual sugar assay in a double-blinded crossover study to evaluate probe excretion and the accuracy of both tests.

Methods: Gastrointestinal permeability was measured in nine volunteers using PEG 400, PEG 1500, and PEG 3350 or lactulose-rhamnose. On 4 separate days, permeability was analyzed after oral intake of placebo or indomethacin, a drug known to increase intestinal permeability. Plasma intestinal fatty acid binding protein and calprotectin levels were determined to verify compromised intestinal integrity after indomethacin consumption. Urinary samples were collected at baseline, hourly up to 5 hours after probe intake, and between 5 and 24 hours. Urinary excretion of PEG and sugars was determined using high-pressure liquid chromatography-evaporative light scattering detection and liquid chromatography-mass spectrometry, respectively.

Results: Intake of indomethacin increased plasma intestinal fatty acid-binding protein and calprotectin levels, reflecting loss of intestinal integrity and inflammation. In this state of indomethacin-induced gastrointestinal compromise, urinary excretion of the three PEG probes and lactulose increased compared with placebo. Urinary PEG 400 excretion, the PEG 3350/ PEG 400 ratio, and the lactulose/rhamnose ratio could accurately detect indomethacin-induced increases in gastrointestinal permeability, especially within 2 hours of probe intake.

Conclusion: Hourly urinary excretion and diagnostic accuracy of PEG and sugar probes show high concordance for detection of indomethacin-induced increases in gastrointestinal permeability. This comparative study improves our knowledge of permeability analysis in man by providing a clear overview of both tests and demonstrates equivalent performance in the current setting.

Keywords: gastrointestinal permeability, polyethylene glycol, dual sugar

\section{Introduction}

Under physiological conditions, the gastrointestinal epithelium provides an effective barrier between the internal and external environment, protecting the body from potentially harmful luminal substances such as bacterial products, digestive enzymes, and antigens. ${ }^{1}$ Gastrointestinal barrier integrity loss is accompanied by an increase in epithelial permeability, reflecting a state in which luminal substances can permeate the barrier and enter the systemic circulation, where they may contribute to a systemic inflammatory response and organ dysfunction. ${ }^{2,3}$ Increased gastrointestinal permeability plays a role in the etiology and worsening of various intestinal and 
systemic diseases, ${ }^{4-7}$ and is considered an important measure of disease activity and prognosis. ${ }^{1}$

Analysis of gastrointestinal permeability is based on the appearance of orally administered probes such as sugars, ${ }^{51}$ chromium-labelled ethylenediamine tetra-acetic acid $\left({ }^{51} \mathrm{CrEDTA}\right)$, and polyethylene glycol (PEG) in the circulation and urine after permeation of the intestinal epithelium. Each probe has its specific advantages and disadvantages, and requires a specific method of detection. ${ }^{1}$

Radioactive ${ }^{51} \mathrm{CrEDTA}$ is easily detectable because it is not naturally present in man. The commonly used dose of $100 \mu \mathrm{Ci}(3.7 \mathrm{Mbq}){ }^{51} \mathrm{CrEDTA}$ is similar to the burden of other clinical nuclear diagnostic tests, and its estimated total radiation dose of $0.12 \mathrm{mSv}^{8}$ is small compared with the worldwide annual background radiation exposure of approximately $3 \mathrm{mSv} .{ }^{9}$ However, ${ }^{51}$ CrEDTA-based permeability tests do expose patients to additional radiation. Therefore, use of ${ }^{51} \mathrm{CrEDTA}$ should be avoided in pediatric patients, women of childbearing age, and in patients requiring multiple permeability analyses. Another concern is that the ${ }^{51} \mathrm{CrEDTA}$ assay is the only test that uses a single probe for permeability analysis; therefore, no correction for the influence of individually determined factors such as intestinal transit is performed.

Sugars provide the opportunity to obtain site-specific information on gastrointestinal permeability when applied in a multi-sugar test solution. ${ }^{1,10}$ Classically, the dual sugar test is used for intestinal permeability analysis. The dual sugar test combines the disaccharide lactulose (molecular weight $342 \mathrm{Da}$ ) with the monosaccharide L-rhamnose (molecular weight $164 \mathrm{Da}$ ). While the exact routes of permeation remain to be clarified, macromolecules such as lactulose are commonly thought to permeate the intestinal mucosa via the size-selective paracellular pathway, while smaller probes such as rhamnose are considered to permeate the mucosa transcellularly as well as paracellularly. ${ }^{1,11,12}$ Various other sugar probes may be added to the permeability test mix, and by exploiting the differences in digestibility and degradation of these probes, permeability assessment can be performed on the gastroduodenal part, small intestine, and large intestine. ${ }^{13,14}$ Furthermore, quantification of plasma sugar concentrations after intake of the sugar test solution enables detection of moderate and transient changes in gastrointestinal permeability. ${ }^{15}$ The use of liquid chromatography-mass spectrometry allows the quantification of very low sugar concentrations, but this technique is expensive and the system is not yet standard laboratory equipment, thereby limiting the applicability of sugar-based permeability analysis.
In addition, sugar permeability analysis may be hampered by the presence of food-derived sugars in urine and plasma, ${ }^{10,16}$ stressing the importance of an adequate analytical technique for accurate permeability analysis.

Another commonly used option for permeability analysis is based on the use of PEG probes of different sizes. While the addition of PEG to some food products, such as artificially sweetened sodas, ${ }^{17,18}$ and its occasional use in clinical practice for colonic lavage limits its applicability, ${ }^{1}$ PEG has a number of advantages over the use of ${ }^{51}$ CrEDTA and sugars for permeability analysis. It does not require radioactivity, is not metabolized by enzymes or degraded by bacteria within the human gastrointestinal tract, ${ }^{1,19,20}$ and the required method of analysis is less expensive and time-consuming than for the other probes. Therefore, PEG probes are suggested to be particularly suitable markers for whole gut permeability assessment in man.

The current lack of consensus for gastrointestinal permeability assessment has led to many studies on intestinal permeability using different probes, making it very difficult to compare and interpret results. Therefore, our aim was to resolve part of this diversity by comparing urinary probe excretion and accuracy of a PEG-based permeability assay with the dual sugar lactulose-rhamnose test in a double-blind crossover study using indomethacin, a nonsteroidal antiinflammatory drug (NSAID), to induce a state of increased gastrointestinal permeability in healthy individuals. ${ }^{21,22}$

\section{Materials and methods}

The current study was approved by the medical ethics committee of Maastricht University Medical Center and was conducted according to the revised version of the Declaration of Helsinki (October 2008, Seoul). The study is part of a larger project registered at the US National Library of Medicine (http://www.clinicaltrials.gov, NCT00943345).

\section{Participants}

Nine healthy men and women (five men, four women; mean age $31.2 \pm 4.7$ years; body mass index $23.9 \pm 0.9$ ) were included in this study. Subjects were not experiencing abdominal complaints during their normal daily activities, as assessed by a questionnaire prior to inclusion. Exclusion criteria were recent (defined as being in the past 30 days) use of any medication, history of abdominal surgery, known hypersensitivity to NSAIDs or food products, pregnancy, alcohol or substance abuse, and any other medical condition that could influence the experimental results (eg, gastrointestinal disease). Volunteers were informed about the nature 
and risks of the experiments. Written consent was obtained at least 5 days before the experiments. No medication use was permitted during the study period.

\section{Pretest restrictions and arrangements}

In the 2 days prior to the experimental test days, test subjects were instructed to maintain normal dietary patterns and to record their dietary intake. In addition, subjects were instructed to avoid alcohol, caffeine, or spicy products, and to maintain diet as recorded for the subsequent test days to minimize the effects of variations in dietary intake. The evening before each test day, participants received a standardized meal (1695 kJ, consisting of $62.6 \mathrm{~g}$ carbohydrate, $18.9 \mathrm{~g}$ protein, and $7.9 \mathrm{~g}$ of fat). All participants maintained normal activities of daily living and refrained from heavy physical activity in the 2 days prior to each test day. Subjects were fasted overnight, but were allowed to drink tap water (maximum of $300 \mathrm{~mL}$ ) in the morning.

\section{Test mix preparation}

The PEG test mix used consisted of $5.7 \mathrm{~g}$ of PEG 400 (Chempri BV, Raamsdonksveer, The Netherlands), $2.5 \mathrm{~g}$ of PEG 1500 (Chempri BV), and $13.8 \mathrm{~g}$ of PEG 3350 (Norgine BV, Amsterdam, The Netherlands), dissolved in $150 \mathrm{~mL}$ of tap water, based on previous studies by Parlesak et al and Kerckhoffs et al. ${ }^{23,24}$ All PEG used in the test mix was tested for safe oral consumption by Basic Pharma BV (Geleen, The Netherlands). The dual sugar mix consisted of $5 \mathrm{~g}$ lactulose (Centrafarm, Etten-Leur, The Netherlands) and $0.5 \mathrm{~g}$ of L-rhamnose (Danisco Sweeteners, Copenhagen, Denmark), also dissolved in $150 \mathrm{~mL}$ of tap water. Both sugars were intended and tested for safe oral human consumption by the manufacturer.

\section{Study design and sampling}

The current study was randomized, controlled, and doubleblind in design. All subjects completed 4 test days. On all these days, gastrointestinal permeability analysis was performed, using either PEG probes or dual sugar probes. Subjects were tested twice using the PEG probes; once to assess permeability under basal (placebo) conditions, and once to assess permeability after indomethacin. On 2 other, separate test days, dual sugar probes were used to assess basal and indomethacin-induced permeability. The interval between test days was at least 7 days, but incidentally extended up to a maximum of 20 days. Subjects received either placebo capsules (to test basal permeability) or capsules with indomethacin (Actavis BV, Baarn, The Netherlands) $75 \mathrm{mg}$ at
$10 \mathrm{pm}$ the night before the test day and $50 \mathrm{mg}$ at 7:30 am ( 1 hour prior to the start of the tests) to increase gastrointestinal permeability. ${ }^{21}$ Capsules were macroscopically identical. Both the participants and investigators were unaware of the content of the capsules. Participants were instructed to drink $200 \mathrm{~mL}$ of tap water per hour during the test.

After ingestion of the probes, urinary samples were collected at baseline and hourly for up to 5 hours after ingestion of the test mix. Urinary volumes were recorded and $4 \mathrm{~mL}$ of each sample was put into a polypropylene tube (Greiner Bio-One, Kremsmünster, Austria) and kept on ice until centrifugation (within 30 minutes after sampling). In addition, subjects collected 5-24-hour urine at home and returned the bottles on the following morning. Urinary volume was recorded, and urine was immediately processed.

Baseline blood samples were collected in prechilled ethylenediamine tetra-acetic acid (EDTA) tubes (BD Vacucontainer, Becton Dickinson Diagnostics, Aalst, Belgium) and immediately centrifuged. Both plasma and urine samples were centrifuged at $4^{\circ} \mathrm{C}$ and $2300 \times \mathrm{g}$ for 15 minutes and stored at $-80^{\circ} \mathrm{C}$ until analysis.

\section{Analysis of intestinal epithelial damage and inflammation}

Intestinal epithelial cell damage was assessed by quantification of plasma concentrations of human intestinal fatty acid binding protein (I-FABP) using a highly specific enzymelinked immunosorbent assay developed inhouse (detection window 20-5000 pg/mL), as previously described. ${ }^{15}$

Inflammation was determined in the same plasma samples by analysis of calprotectin concentrations using a commercially available calprotectin enzyme-linked immunosorbent assay (detection window $0.78-50 \mathrm{ng} / \mathrm{mL}$ ) kindly provided by Hycult Biotechnology (Uden, The Netherlands). Both markers were determined on all experimental days, in each first blood sample of the day $(t=0)$.

\section{Analysis of permeability probes}

All chemicals used for laboratory purposes were of analytical grade, and unless specified otherwise, were purchased from Sigma Aldrich (St Louis, MO). Ultrapure water was generated through a Super-Q water purification system (Millipore, Billerica, MA) and used as the source of water for all analytical assays.

Urinary sugar concentrations were determined by isocratic ion-exchange chromatography in combination with mass spectrometry detection, as previously described. ${ }^{10}$ Urinary PEG concentrations were analyzed by reversed 
phase high-pressure liquid chromatography in combination with evaporative light scatter detection. The analysis was based on PEG analysis as described by Kerckhoffs et al. ${ }^{24}$ In short, samples were thawed to room temperature and transferred to $1.5 \mathrm{~mL}$ Eppendorf cups containing $6 \mathrm{mg}$ of 5 -sulfosalicylic acid. After thorough mixing, samples were centrifuged at $50,000 \times \mathrm{g}$ for 10 minutes at $4^{\circ} \mathrm{C}$ in a highspeed centrifuge (Model Biofuge Stratos; Heraeus, Hanau, Germany). Clear urinary supernatant was injected into the high-pressure liquid chromatography system (2 Model PU-980 pumps; Jasco, Easton, MD) using a WISP auto sampler (Model 715; Waters, Milford, MA). Separation of PEG was achieved by reversed-phase chromatography on an Allsphere ODS- 2 column (particle size $3 \mu \mathrm{M}, 150 \times 4.6 \mathrm{~mm}$ ID; Grace Alltech, Deerfield, IL), mounted in a Mistral column oven (Separations Analytical Instruments, Ambacht, The Netherlands) set to $50^{\circ} \mathrm{C}$. The simplified workup of the urinary samples, using only high-speed centrifugation to prepare the urinary samples, did not lead to accelerated degradation of the Allsphere column. Solvents used were water (solvent A) and methanol/water ( $90 / 10 \mathrm{v} / \mathrm{v}$; solvent B). The gradient of the mobile phase used for separation of the PEG probes was: 0 minutes, $95 \%$ solvent A; 9 minutes, $95 \%$ solvent $\mathrm{A} ; 10$ minutes, $70 \%$ solvent $\mathrm{A} ; 11$ minutes, $45 \%$ solvent A; 12 minutes, $45 \%$ solvent $\mathrm{A} ; 13$ minutes, $20 \%$ solvent $\mathrm{A}$; 14 minutes, $10 \%$ solvent $\mathrm{A} ; 15$ minutes, $10 \%$ solvent $\mathrm{A}$; and 16 minutes, $0 \%$ solvent A. PEG detection was performed using evaporative light scatter detection on a model 2000 ES detector (Grace Alltech). Optimal signal-to-noise ratios for PEG determination were obtained by setting the evaporative light scatter detector to a temperature of $50^{\circ} \mathrm{C}$ with a nitrogen gas flow of $1.5 \mathrm{~mL} /$ minute at 5 bar, and with the impactor off, while maintaining a gradient flow of $1.00 \mathrm{~mL} /$ minute.

PEG concentrations in samples were determined using external standard calibration. Analytical standards were prepared by dissolving analytical grade PEG 400, PEG 1500 , and PEG 3350 in water, after which aliquots of the standard were stored at $-80^{\circ} \mathrm{C}$. Standards were run before each set of 10 samples. PEG 400 concentration was measured by injecting $2 \mu \mathrm{L}$ of sample into the detector, and for optimal detection of the lower concentrated PEG 1500 and PEG 3350, the injection volume was increased to a maximum of $100 \mu \mathrm{L}$. If urinary probe concentrations in the samples collected at baseline were above zero, these baseline levels were subtracted from the concentrations measured in urinary samples collected at subsequent time points. Urinary excretions of sugars and PEG were calculated by multiplying urinary concentrations with urine volume. Urinary recovery of the orally ingested probe dose was calculated and expressed as a percentage of the oral dose. Probe ratios were calculated from the urinary excretion values.

Urine spiked with PEG 400 was analyzed before and after addition of indomethacin, and urinary samples containing indomethacin were spiked with PEG 400 to confirm our hypothesis that the presence of indomethacin (molecular weight $358 \mathrm{Da}$ ) did not interfere with PEG 400 analysis in urine.

\section{Statistical analysis}

Statistical analysis was performed using GraphPad Prism (version 5; GraphPad, San Diego, CA). Normality of all data was verified using the Kolmogorov-Smirnov test. Measurements of basal intestinal permeability and permeability after indomethacin intake were compared using the Wilcoxon signed-rank test, considering the small number of study participants. For the PEG assay and the dual sugar permeability test, receiver operating characteristic curves were plotted to determine the cutoff points and discriminate between basal and indomethacin-induced intestinal permeability in our healthy participants. The ideal cutoff point, defined as the maximum sum of sensitivity and specificity, was reported. Correlations were computed by calculating Spearman correlation coefficients $\left(r_{S}\right)$, unless specifically stated otherwise. Data from nine healthy individuals with and without intake of indomethacin are presented as the mean \pm standard error of the mean if most data were normally distributed, or as the median (range) if not. $P<0.05$ was considered to be statistically significant.

\section{Results PEG analysis: linearity, accuracy, and limit of detection}

Linearity of PEG detection was determined by injecting a standard containing the three PEG probes. Calibration curves were linear in a range from 0 to $200 \mu \mathrm{M}$. The results of linear regression analysis of the calibration curves are shown in Table 1. The lower limit of quantification observed at a signal-to-noise ratio of 5:1 was $0.63 \mu \mathrm{M}$ for PEG 400, $0.14 \mu \mathrm{M}$ for PEG 1500, and $0.054 \mu \mathrm{M}$ for PEG 3350. Coefficients of variation were calculated from 10 measurements of urinary samples obtained from different individuals. These samples were spiked with different PEG concentrations ranging from $0 \mu \mathrm{L}$ to $100 \mu \mathrm{L}$, resulting in overall coefficients of variation of $3.6 \%$ for PEG 400, 2.7\% for PEG 1500, and 1.2\% for PEG 3350. 
Table I Slope, intercept, and determination coefficient $\left(R^{2}\right)$ of linear regression analysis of calibration curves for PEG 400, PEG I500, and PEG 3350

\begin{tabular}{lllll}
\hline & Slope $^{\mathrm{a}}$ & Y intercept $^{\mathrm{a}}$ & $\mathbf{X}$ intercept & $\mathbf{R}^{\mathbf{2}}$ \\
\hline PEG 400 & $8.761 \mathrm{e}+006 \pm$ & $-95362 \pm 43874$ & 0.01088 & 0.9816 \\
& 489706 & & & \\
PEG 1500 & $7.846 \mathrm{e}+007 \pm$ & $-743532 \pm 310682$ & 0.00948 & 0.9884 \\
& $3.468 \mathrm{e}+006$ & & & \\
PEG 3350 & $1.955 \mathrm{e}+008 \pm$ & $-1.718 \mathrm{e}+006 \pm$ & 0.00879 & 0.9754 \\
& $1.388 \mathrm{e}+007$ & 816305 & & \\
\hline
\end{tabular}

Note: a ${ }^{D}$ ata are presented as the mean \pm standard deviation.

Abbreviations: PEG, polyethylene glycol; h, hour.

\section{Indomethacin induces loss of gastrointestinal barrier integrity}

To verify the putative loss of gastrointestinal barrier integrity induced by indomethacin, plasma I-FABP and calprotectin were analyzed. I-FABP levels, determined in plasma samples collected at baseline (ie, before intake of permeability probes), were significantly higher after intake of indomethacin (443 [206-1306] pg/mL) compared with placebo $(336$ [76-885] $\mathrm{pg} / \mathrm{mL}, P<0.01$, Figure $1 \mathrm{~A})$, reflecting minor intestinal injury in the indomethacin-challenged situation. In line with this, plasma calprotectin levels increased after intake of indomethacin (116 [28-273] ng/mL), compared with placebo (76 [26-138] ng/mL, $P=0.0005$, Figure 1B). While none of the volunteers experienced gastrointestinal complaints during the test days or in the week thereafter, these plasma data confirm the putative loss of a small amount of intestinal integrity and inflammation in the indomethacinchallenged situation.

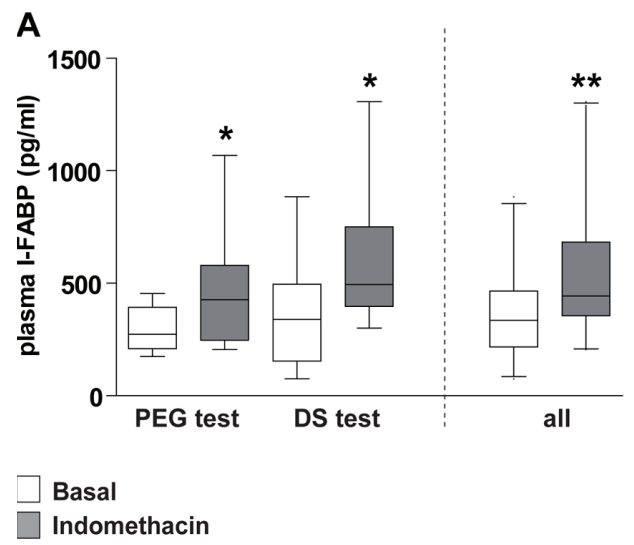

\section{Indomethacin-induced permeability changes assessed by PEG probes}

Indomethacin increases urinary PEG probe excretion Urinary PEG 400 excretion increased significantly after indomethacin intake compared to basal (placebo) conditions in the 0-1-hour, 0-2-hour, and 0-5-hour urinary collections (Figure 2A and B, Table 2), and a trend towards increased 0-24-hour PEG 400 excretion was observed $(P=0.06$, Figure 2A). In line with PEG 400 data, urinary excretion of PEG 1500 increased significantly after intake of indomethacin in the 0-2-hour and 0-5-hour urinary collections. No differences in 0-24-hour PEG 1500 excretion were observed between placebo and the indomethacin-challenged state (Figure $2 \mathrm{C}$ and D; Table 2). Urinary excretion of the largest probe, PEG 3350, significantly increased after indomethacin intake only in the 0-5-hour urinary collection (Figure 2E and F, Table 2).

Interestingly, two of the nine individuals consistently showed decreased 0-2-hour urinary PEG excretion in the indomethacin-challenged state (Figure 2B, D, and F). Decreased plasma I-FABP levels (454 pg/mL at baseline to $433 \mathrm{pg} / \mathrm{mL}$ after indomethacin intake) corroborated the decreased PEG excretion in one of these individuals, suggesting a lack of indomethacin-induced epithelial integrity loss. The other subject had increased plasma I-FABP levels but decreased PEG probe excretion after intake of indomethacin.

Statistical analysis of the PEG excretion rates revealed that early urinary collections can be used to detect increased permeability with acceptable sensitivity and specificity (Table 3). Alternatively, 0-5-hour urinary PEG excretion may be used to detect increased gastrointestinal permeability after intake of indomethacin (Table 3 ).

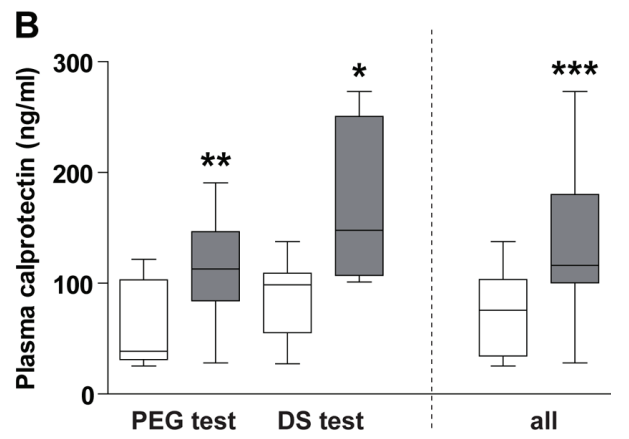

Figure I Intake of indomethacin results in minor intestinal cell damage and inflammation. (A) Plasma I-FABP levels increase after intake of indomethacin, indicating indomethacin-induced enterocyte damage. (B) Plasma calprotectin levels increase after intake of indomethacin, indicating inflammation after indomethacin ingestion. Notes: Data are shown as the median (range) of baseline plasma samples $(t=0)$ of nine healthy individuals. $* P<0.05$; $* * P<0.0 \mathrm{I}$; $* * * P=0.0005$, significantly different from basal (placebo) conditions.

Abbreviations: PEG, polyethylene glycol; DS, dual sugar; I-FABP, intestinal fatty acid binding protein. 

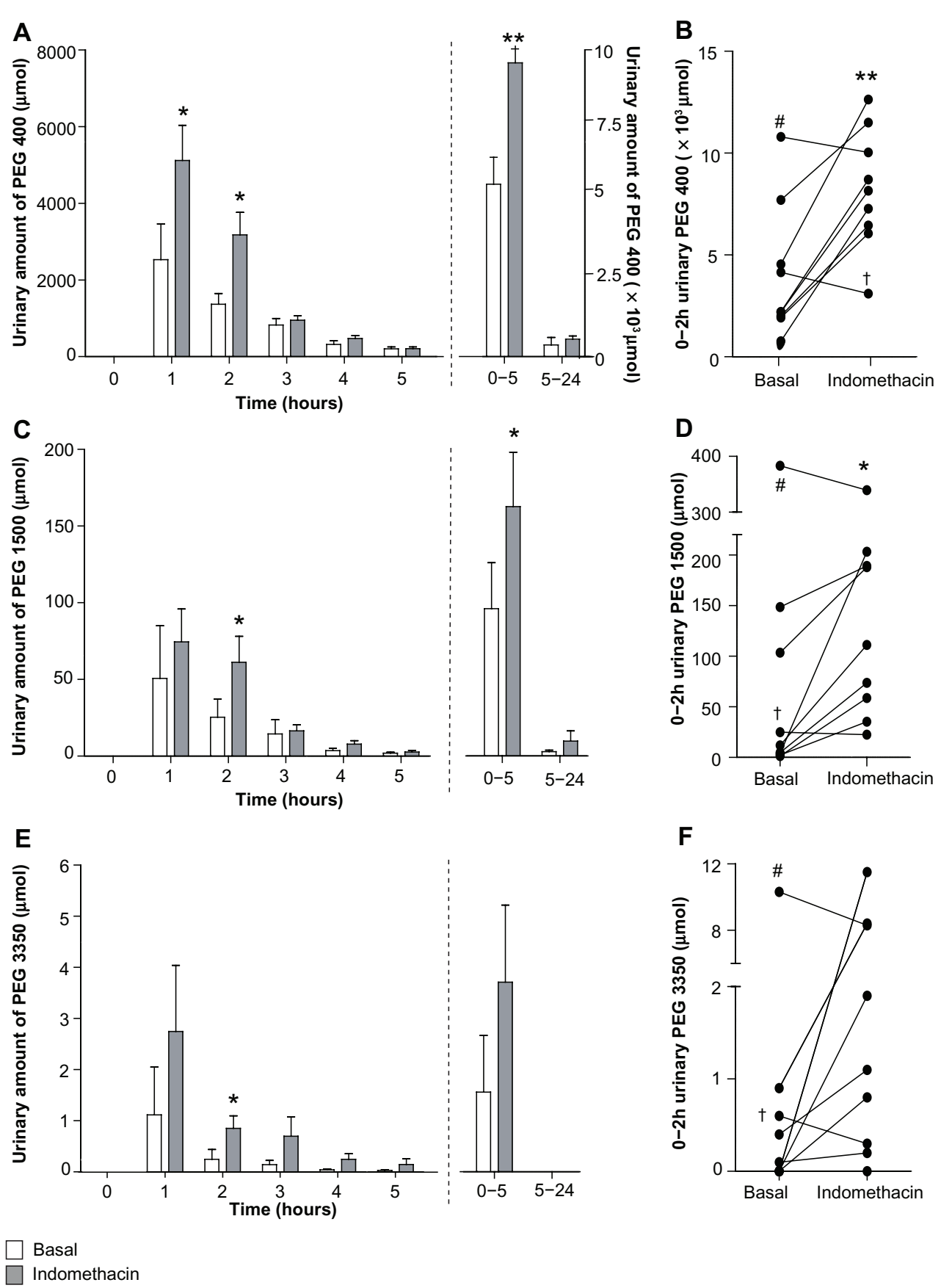

Figure 2 Urinary excretion of PEG probes increases after intake of indomethacin. (A) Urinary PEG 400 excretion is elevated after intake of indomethacin. (B) Individual 0-2-hour urinary PEG 400 excretion from nine healthy individuals in the basal and indomethacin-challenged state. (C) Indomethacin increases urinary excretion of PEG I500. (D) Individual 0-2-hour urinary PEG I500 excretion from nine healthy individuals in the basal and indomethacin-challenged state. (E) Urinary excretion of PEG 3350 tends to increase after indomethacin. (F) 0-2-hour urinary PEG 3350 excretion is increased in the majority of volunteers.

Notes: Data are shown as the mean \pm standard error of the mean of nine healthy individuals, unless otherwise stated. In two volunteers, indicated as ${ }^{\dagger}$ and ${ }^{\#}$ respectively, decreased urinary PEG excretion was observed for each individual PEG probe, while one individual had excessively high indomethacin-induced PEG 3350 excretion in 3-hour and $0-5$-hour urine and was therefore not included in Figure $2 \mathrm{E}$. $* \mathrm{P}<0.05 ; * * \mathrm{P}<0.0 \mathrm{I}$, significantly different from basal (placebo) conditions.

Abbreviation: PEG, polyethylene glycol.

To increase our understanding of the relationship between cellular injury and increased gastrointestinal permeability, correlations between intestinal cell integrity loss (ie, plasma I-FABP levels) and increased gastrointestinal permeability (ie, urinary PEG excretion) were sought. Normalized levels of I-FABP, a small $14-15 \mathrm{kDa}$ protein ${ }^{25}$ measured in plasma samples collected just before probe intake, correlated significantly with $\mathrm{PEG} 3350$ excretion $\left(\mathrm{R}_{\mathrm{S}}=0.51, P<0.05\right.$ for 0-2-hour urinary excretion and $\mathrm{R}_{\mathrm{S}}=0.69, P<0.005$ for $0-5$-hour urinary excretion), while no correlations were 
Table 2 Urinary PEG probe excretion in basal (placebo) and indomethacin-challenged state

\begin{tabular}{|c|c|c|c|c|c|}
\hline & \multicolumn{2}{|c|}{$\begin{array}{l}\text { Basal probe excretion } \\
(\mu \mathrm{mol})\end{array}$} & \multicolumn{2}{|c|}{$\begin{array}{l}\text { Indomethacin probe excretion } \\
(\mu \mathrm{mol})\end{array}$} & \multirow[t]{2}{*}{$P$} \\
\hline & Median & Range & Median & Range & \\
\hline PEG 400 (0-I-h urine) & 1487 & $(108-8260)$ & 5795 & (I272-9923) & $<0.05$ \\
\hline PEG 400 (0-2-h urine) & 1969 & $(458-11,013)$ & 8230 & $(2908-12,942)$ & $<0.01$ \\
\hline PEG 400 (0-5-h urine) & 4597 & $(208 I-12,123)$ & 9893 & $(4 \mid 80-14,808)$ & $<0.01$ \\
\hline PEG I500 (0-I-h urine) & 3.20 & $(0.40-318.30)$ & 35.90 & $(6.70-165.90)$ & 0.20 \\
\hline PEG I500 (0-2-h urine) & 11.90 & $(1.30-383.30)$ & 111.00 & $(22.60-339.60)$ & $<0.05$ \\
\hline PEG I500 (0-5-h urine) & 18.90 & $(2.80-386.50)$ & 174.20 & $(35.50-366.10)$ & 0.05 \\
\hline PEG 3350 (0-I-h urine) & 0.00 & $(0.00-8.60)$ & 0.40 & $(0.00-9.80)$ & 0.67 \\
\hline PEG 3350 (0-2-h urine) & 0.10 & $(0.00-10.30)$ & 1.10 & $(0.00-11.50)$ & 0.20 \\
\hline PEG 3350 (0-5-h urine) & 0.40 & $(0.00-10.30)$ & 1.80 & $(0.00-104.00)$ & $<0.05$ \\
\hline
\end{tabular}

Abbreviations: PEG, polyethylene glycol; h, hour.

found between I-FABP and other PEG probes. Hence, urinary PEG 3350 excretion correlates with increased I-FABP levels after indomethacin consumption, suggesting a role for loss of enterocyte integrity and subsequent release of cytosolic protein I-FABP in the development of intestinal barrier loss reflected by increased PEG 3350 permeation.

\section{Indomethacin and urinary PEG probe ratios}

Gastrointestinal permeability can be assessed either by determination of urinary probe excretion or by calculation of probe ratios. ${ }^{1}$ Expressing permeability information as the ratio of a larger probe over a smaller probe has a couple of advantages compared with the use of single-probe urinary excretion rates. ${ }^{1}$ Most importantly, permeability probe ratios are less affected by individually determined factors unrelated to gastrointestinal permeability, such as renal clearance. ${ }^{26}$ Therefore, the three PEG probes were also analyzed as urinary PEG ratios. The PEG ratios determined in the 0-2-hour urinary collections are shown in Figure 3. While the majority of individuals showed an increase in all

Table 3 Overall accuracy and cutoff points of both urine-based permeability tests

\begin{tabular}{|c|c|c|c|c|c|}
\hline Urinary collection & $\begin{array}{l}\text { Cutoff point } \\
(\mu \mathrm{mol})\end{array}$ & Sensitivity & Specificity & AUC $(95 \% \mathrm{Cl})$ & $P$ \\
\hline \multicolumn{6}{|l|}{ PEG 400} \\
\hline $0-I-h$ & 2288 & $89 \%$ & $67 \%$ & $0.79(0.57-1.01)$ & $<0.05$ \\
\hline $\mathrm{I}-2-\mathrm{h}$ & 1507 & $100 \%$ & $67 \%$ & $0.85(0.67-1.03)$ & 0.01 \\
\hline $0-2-h$ & 5226 & $89 \%$ & $78 \%$ & $0.84(0.65-1.03)$ & $<0.05$ \\
\hline $0-5-h$ & 7251 & $89 \%$ & $78 \%$ & $0.83(0.63-1.03)$ & $<0.05$ \\
\hline \multicolumn{6}{|l|}{ PEG I500 } \\
\hline $0-I-h$ & 5.00 & $100 \%$ & $56 \%$ & $0.74(0.49-0.99)$ & 0.09 \\
\hline $\mathrm{I}-2-\mathrm{h}$ & 9.00 & $100 \%$ & $67 \%$ & $0.79(0.56-1.01)$ & $<0.05$ \\
\hline $0-2-h$ & 20.00 & $100 \%$ & $56 \%$ & $0.77(0.53-1.01)$ & 0.06 \\
\hline $0-5-h$ & 33.00 & $100 \%$ & $67 \%$ & $0.74(0.48-1.00)$ & 0.09 \\
\hline \multicolumn{6}{|l|}{ PEG 3350} \\
\hline $0-I-h$ & 0.05 & $78 \%$ & $56 \%$ & $0.64(0.38-0.90)$ & 0.31 \\
\hline $\mathrm{I}-2-\mathrm{h}$ & 0.15 & $78 \%$ & $78 \%$ & $0.77(0.53-1.00)$ & 0.06 \\
\hline $0-2-h$ & 0.15 & $89 \%$ & $56 \%$ & $0.74(0.50-0.98)$ & 0.09 \\
\hline $0-5-h$ & 0.75 & $89 \%$ & $78 \%$ & $0.78(0.54-1.02)$ & $<0.05$ \\
\hline \multicolumn{6}{|l|}{ PEG 3350/400 } \\
\hline $0-I-h$ & $5 e-005$ & $67 \%$ & $89 \%$ & $0.77(0.54-1.00)$ & 0.05 \\
\hline I-2-h & $5 e-005$ & $78 \%$ & $89 \%$ & $0.79(0.55-1.03)$ & $<0.05$ \\
\hline $0-2-h$ & $5 e-005$ & $78 \%$ & $89 \%$ & $0.80(0.58-1.03)$ & $<0.05$ \\
\hline $0-5-h$ & $5 e-005$ & $89 \%$ & $89 \%$ & $0.85(0.66-1.06)$ & 0.01 \\
\hline \multicolumn{6}{|l|}{ L/R ratio } \\
\hline $0-I-h$ & 0.17 & $75 \%$ & $100 \%$ & $0.85(0.62-1.09)$ & $<0.05$ \\
\hline $\mathrm{I}-2-\mathrm{h}$ & 0.105 & $78 \%$ & $89 \%$ & $0.86(0.68-1.04)$ & $<0.05$ \\
\hline $0-2-h$ & 0.13 & $78 \%$ & $100 \%$ & $0.87(0.69-1.07)$ & $<0.05$ \\
\hline $0-5-h$ & 0.18 & $78 \%$ & $78 \%$ & $0.77(0.65-1.08)$ & 0.08 \\
\hline
\end{tabular}

Abbreviations: AUC, area under the curve; $\mathrm{Cl}$, confidence interval; PEG, polyethylene glycol; h, hour. 

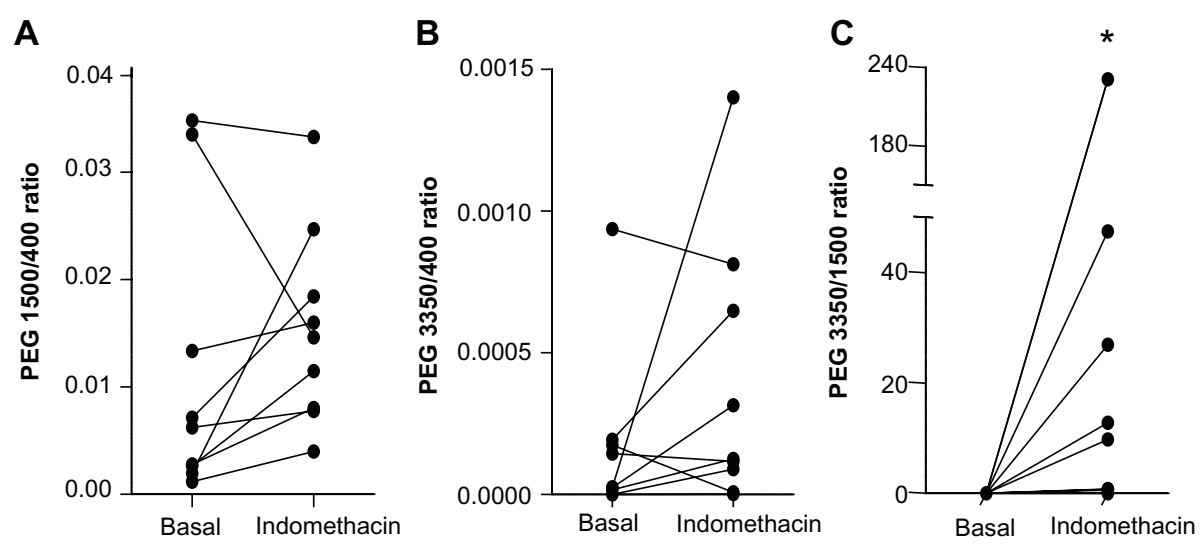

Figure 3 Changes in PEG probe ratios after intake of indomethacin. (A) The majority of volunteers had an increased 0-2-hour urinary PEG I500/PEG 400 ratio after indomethacin intake. (B) Indomethacin resulted in an increased urinary PEG 3350/PEG 400 ratio in most individuals. (C) Individual 0-2-hour urinary PEG 3350/PEGI500 ratios increased after indomethacin intake.

Note: $* P<0.05$, significantly different from basal (placebo) conditions.

Abbreviation: PEG, polyethylene glycol.

three urinary PEG ratios after consumption of indomethacin (Figure 3), statistical significance was not reached. In line with these data, urinary PEG ratios did not correlate with plasma I-FABP levels. Only the PEG 3350/PEG 400 ratio increased significantly after intake of indomethacin compared with basal conditions ( $P \leq 0.05$ in $0-1$-hour, $2-3$-hour, and $0-5$-hour urine collections).

Consistent with these results, the statistical analysis shows that only the urinary PEG 3350/PEG 400 ratio could accurately detect indomethacin-induced increases in gastrointestinal permeability (Table 3 ).

\section{Indomethacin-induced permeability changes assessed by the dual sugar test}

The urinary excretion of the $342 \mathrm{Da}$ sugar probe lactulose increased after intake of indomethacin, an increase that was most pronounced in the first 2 hours after intake of the sugars (Figure 4A and B). Urinary recovery in these first 2 hours was roughly $0.110 \% \pm 0.030 \%$ after intake of indomethacin compared with $0.096 \% \pm 0.024 \%$ under basal conditions. Two individuals had decreased lactulose excretion in the indomethacin-challenged state, but these individuals were not the same individuals who showed decreased PEG excretion after indomethacin intake.

Urinary excretion of the 164 Da sugar probe rhamnose decreased after intake of indomethacin, reaching significance at the $0-2$-hour and $0-5$-hour urinary collections $(P<0.01$ and $P<0.05$, respectively, Figure $4 \mathrm{C}$ and $\mathrm{D}) ; 0-5$-hour urinary rhamnose excretion levels were equivalent to $8.6 \% \pm 0.8 \%$ and $6.4 \% \pm 0.5 \%$ urinary recovery after placebo and indomethacin intake, respectively $(P<0.05)$. Due to the increased lactulose excretion and simultaneous decrease in rhamnose excretion, urinary ratios of the large lactulose probe and the smaller rhamnose probe increased significantly after indomethacin intake (Figure 4E and F). In keeping with the excretion profiles of lactulose and the PEG probes, this increase was most pronounced in the first 2 hours after probe intake (Figure 4E and F). While lactulose excretion could not provide accurate sensitivity and specificity to detect indomethacin-induced intestinal permeability as a single probe, urinary $\mathrm{L} / \mathrm{R}$ ratios in these first 2 hours could accurately detect increased permeability in the indomethacin-challenged state (Table 3).

\section{Discussion}

Intestinal permeability is an important parameter of gut barrier function, but the availability of multiple permeability tests impedes the interpretation and comparison of urinary probe excretion. The aim of the current study was to compare urinary probe excretion and accuracy of a PEG assay and a dual sugar lactulose-rhamnose permeability assay in a double-blind crossover study using the NSAID, indomethacin, to induce increased gastrointestinal permeability.

Before performing the human study, the time-consuming sample preparation protocol for analysis of the PEG probes was optimized. Analysis of PEG probes in biological samples was previously described by Parlesak et al using highpressure liquid chromatography with differential refraction index detection. ${ }^{23}$ We enhanced the analytical sensitivity of PEG analysis using evaporative light scatter detection, as described by Kerckhoffs et al, ${ }^{24}$ while simultaneously reducing the sample preparation protocol to a single sample centrifugation step prior to high-pressure liquid chromatography analysis. Due to this simple high-speed centrifugation step, the time-consuming extraction methods previously 
A
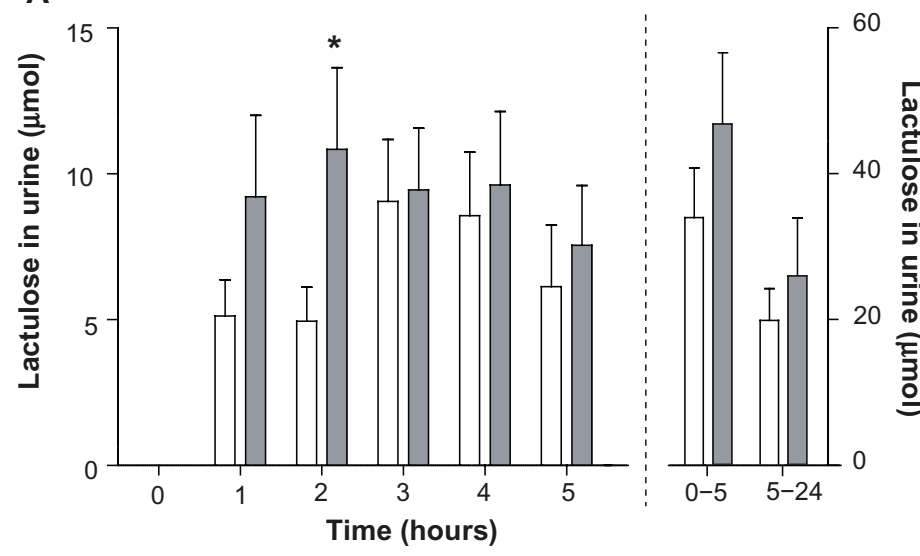

C

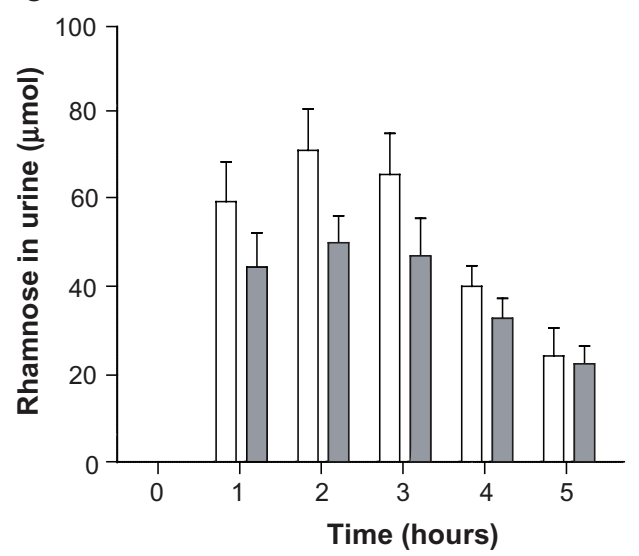

E

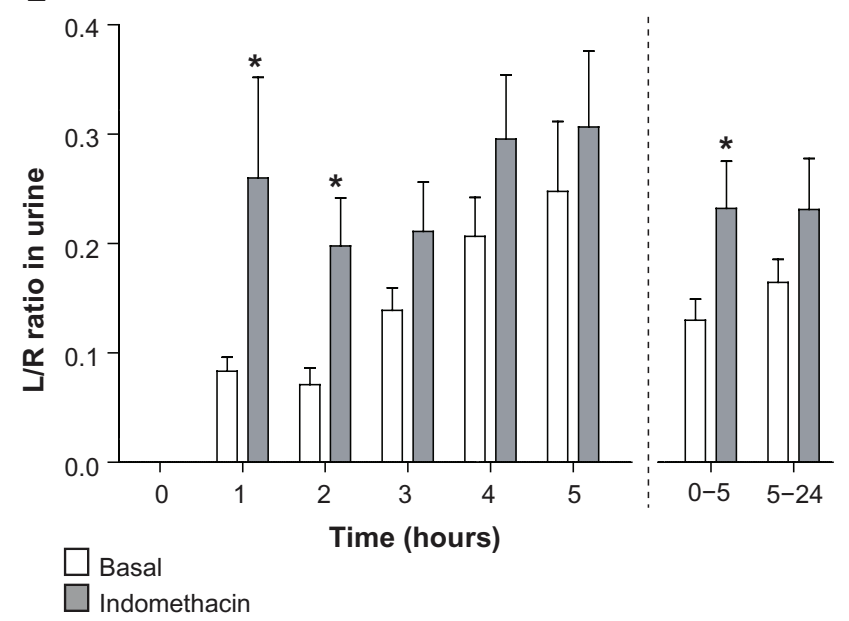

B

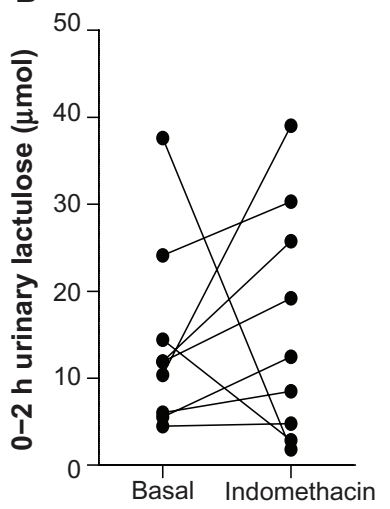

D

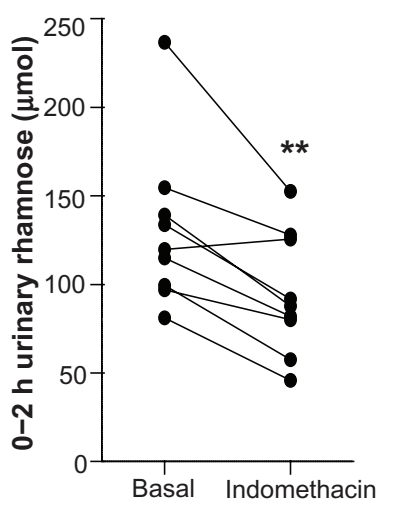

F

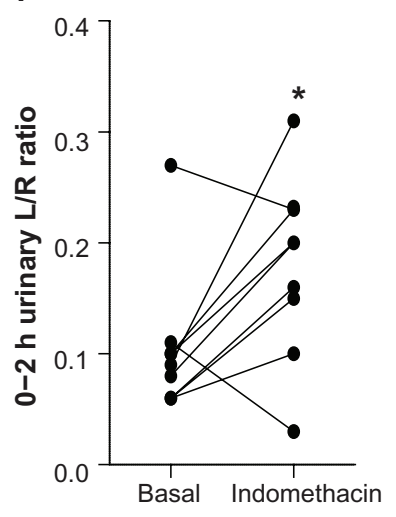

Figure 4 Urinary excretion of sugar probes increases after intake of indomethacin. (A) Urinary excretion of lactulose tended to increase after intake of indomethacin. (B) Individual 0-2-hour urinary lactulose excretion of nine healthy individuals in basal and indomethacin-challenged state. (C) Rhamnose excretion decreased after intake of indomethacin. (D) 0-2-hour urinary rhamnose excretion of nine healthy individuals decreased significantly in the indomethacin-challenged state. (E) Urinary L/R ratio increased after intake of indomethacin, indicating increased intestinal permeability. (F) Individual 0-2-hour urinary L/R ratios are increased after indomethacin intake. Notes: Data are shown as the mean \pm standard error of the mean of nine healthy individuals. $* P<0.05$; $* * P<0.0$, significantly different from basal (placebo) conditions.

required to obtain satisfactory lower limits of detection were no longer necessary, ${ }^{23,24}$ making PEG analysis rapid and simple. Sugar analysis was performed by high-pressure liquid chromatography-mass spectrometry detection, as described elsewhere. ${ }^{10}$ The human study was performed to analyze the application of this optimized PEG approach in a controlled experimental setting and to compare its results with data obtained using the classical dual sugar test.

Increased gastrointestinal permeability was previously described after short-term consumption of indomethacin. ${ }^{21}$ 
Because indomethacin was used in the current study as a method to increase gastrointestinal permeability, we first verified the putative loss of gastrointestinal barrier integrity in our participants. Indeed, significantly increased plasma levels of I-FABP and calprotectin were observed after indomethacin intake, allowing the use of this setting for controlled evaluation of the PEG test and the dual sugar test for analysis of gastrointestinal permeability.

In this experimental setting of integrity loss, urinary excretion of PEG 400, PEG 1500, and PEG 3350 increased after indomethacin consumption. While the absolute excretion rates of the three different-sized PEG probes varied, the urinary excretion profiles of the PEG probes showed high consistency. Urinary excretion of lactulose, the 342 Da sugar probe, increased in the indomethacin-challenged situation in a similar fashion. Indomethacin-induced increases in urinary excretion of PEG and lactulose were most pronounced in the first 2 hours after probe intake. The latter suggests the presence of increased upper gastrointestinal permeability after indomethacin intake, which correlates with the established injury profile of NSAIDs, especially affecting the upper gastrointestinal tract and small intestine. ${ }^{27}$

It is thought that urinary permeability ratios of two different-sized probes provide more reliable information on gastrointestinal permeability than single probe excretion rates for one-time analysis. ${ }^{1}$ The main reason for this assumption is that urinary probe ratios are less affected by everyday variations in urinary output due to gastric emptying and intestinal transit. ${ }^{1}$ In the current study, both the PEG probes and the sugar probes are expressed as urinary ratios and as single probe excretion rates. PEG probes are not commonly expressed as excretion ratios, ${ }^{28,29}$ and since the excretion of all three PEG probes increased after indomethacin consumption, the increase in urinary PEG ratios was not very pronounced in this study. Only the PEG 3350/PEG 400 ratio could detect increased gastrointestinal permeability after indomethacin use with accurate sensitivity and specificity. Compared with the $\mathrm{PEG}$ ratios, the urinary $\mathrm{L} / \mathrm{R}$ ratio increased more distinctly, especially in the first 2 hours after probe intake, due to an indomethacin-induced increase in lactulose excretion and a simultaneous decrease in urinary rhamnose excretion. The standing theory is that large $(>300 \mathrm{Da})$ probes permeate the gastrointestinal mucosa in a paracellular manner, while smaller probes, such as rhamnose, travel via both paracellular and transcellular routes. ${ }^{30}$ This theory dictates that the PEG-based permeability assay provides information on the paracellular permeability pathway, ${ }^{31}$ while the dual sugar test assesses both the paracellular and transcellular pathway.
However, because diffusion of water also occurs transcellularly, a hyperosmolar luminal content, such as the $5 \mathrm{~g}$ lactulose dose given in the current study, may affect the permeability of small, transcellular traveling probes by directing the water and small probe (ie, rhamnose) flux towards the hyperosmolar intestinal lumen. ${ }^{32}$ It remains to be clarified whether changes in osmolarity affect the reliability of the permeability test.

Our aim was to compare urinary probe excretion as well as the accuracy of both tests. The main finding of this study was that the urinary excretion of large $(>300 \mathrm{Da})$ probes, urinary probe ratios, and accuracy of the tests are very much alike. Single PEG 400 excretion, the PEG 3350/ PEG 400 ratio, and the $L / R$ ratio determined in 0-2-hour urine after probe intake all provided accurate detection of indomethacin-induced increases in gastrointestinal permeability. The L/R cutoff point of 0.18 in $0-5$-hour urine is in line with data from previous studies using the dual sugar test after short-term indomethacin consumption ${ }^{33,34}$ and prolonged indomethacin intake. ${ }^{35-37}$ Unfortunately, no cutoff points were reported in previous studies using PEG for permeability analysis in healthy individuals after indomethacin. Previously, Bjarnason et al studied the intestinal permeability of healthy subjects and patients with celiac disease with PEG 400, lactulose, rhamnose, and ${ }^{51} \mathrm{CrEDTA}$, and found similar excretion and sensitivity rates for both the dual sugar test and ${ }^{51}$ CrEDTA permeability test. ${ }^{38}$ However, PEG 400 excretion was decreased in the latter study in the event of increased permeability due to celiac disease. ${ }^{38}$ We have to conclude that due to the numerous factors influencing urinary probe excretion, cutoff points, and accuracy data, one needs to evaluate the different permeability tests in one study in order to enable a correct comparison of test performance.

Data from the current study increase our knowledge about permeability probe excretion by giving a clear overview of urinary PEG and sugar probe excretion over time in a controlled, experimental setting. Future studies are warranted to evaluate the applicability of the permeability tests in clinical settings, eg, for permeability assessment in patients with intestinal diseases, such as celiac disease. Based on the current findings, we consider that both tests may be used to assess gastrointestinal permeability, using urinary probe ratios if multiple testing is impossible, such as in patients with active disease. Furthermore, we recommend combining gastrointestinal permeability assessment with the analysis of plasma I-FABP and calprotectin levels, providing a more thorough overview of gastrointestinal mucosal integrity. 
PEG analysis may provide information on whole-gut permeability, while the dual sugar assay requires additional inert sugars in the test mix to enable analysis of permeability in the large intestine. ${ }^{1}$

In conclusion, the current study demonstrates that gastrointestinal permeability tests based on the urinary excretion of PEG and lactulose-rhamnose show equivalent performance in healthy individuals after NSAID consumption. Given that a clear demand for a standardized whole gut permeability test continues to exist, efforts to develop the ideal permeability test should be continued.

\section{Disclosure}

The authors report no conflicts of interest in this work.

\section{References}

1. Bjarnason I, MacPherson A, Hollander D. Intestinal permeability: an overview. Gastroenterology. 1995;108(5):1566-1581.

2. Fink MP, Delude RL. Epithelial barrier dysfunction: a unifying theme to explain the pathogenesis of multiple organ dysfunction at the cellular level. Crit Care Clin. 2005;21(2):177-196.

3. Chang M, Kistler EB, Schmid-Schonbein GW. Disruption of the mucosal barrier during gut ischemia allows entry of digestive enzymes into the intestinal wall. Shock. 2012;37(3):297-305.

4. Heyman M, Abed J, Lebreton C, Cerf-Bensussan N. Intestinal permeability in coeliac disease: insight into mechanisms and relevance to pathogenesis. Gut. September 2, 2011. [Epub ahead of print.]

5. Katz KD, Hollander D, Vadheim CM, et al. Intestinal permeability in patients with Crohn's disease and their healthy relatives. Gastroenterology. 1989;97(4):927-931.

6. Zuckerman MJ, Watts MT, Bhatt BD, Ho H. Intestinal permeability to [51Cr]EDTA in infectious diarrhea. Dig Dis Sci. 1993;38(9): 1651-1657.

7. Besselink MG, van Santvoort HC, Renooij W, et al. Intestinal barrier dysfunction in a randomized trial of a specific probiotic composition in acute pancreatitis. Ann Surg. 2009;250(5):712-719.

8. Davies NM. Review article: non-steroidal anti-inflammatory druginduced gastrointestinal permeability. Aliment Pharmacol Ther. 1998;12(4):303-320.

9. Zeng W. Communicating radiation exposure: a simple approach. J Nucl Med Technol. 2001;29(3):156-158.

10. van Wijck K, van Eijk HM, Buurman WA, Dejong CH, Lenaerts K. Novel analytical approach to a multi-sugar whole gut permeability assay. J Chromatogr B Analyt Technol Biomed Life Sci. 2011;879(26): 2794-2801.

11. Fihn BM, Sjoqvist A, Jodal M. Permeability of the rat small intestinal epithelium along the villus-crypt axis: effects of glucose transport. Gastroenterology. 2000;119(4):1029-1036.

12. Van Itallie CM, Holmes J, Bridges A, et al. The density of small tight junction pores varies among cell types and is increased by expression of claudin-2. J Cell Sci. 2008;121(Pt 3):298-305.

13. Meddings JB, Gibbons I. Discrimination of site-specific alterations in gastrointestinal permeability in the rat. Gastroenterology. 1998;114(1): 83-92.

14. Meddings JB, Sutherland LR, Byles NI, Wallace JL. Sucrose: a novel permeability marker for gastroduodenal disease. Gastroenterology. 1993;104(6):1619-1626.

15. van Wijck K, Lenaerts K, van Loon LJ, Peters WH, Buurman WA, Dejong $\mathrm{CH}$. Exercise-induced splanchnic hypoperfusion results in gut dysfunction in healthy men. PLoS One. 2011;6(7): e22366.
16. Cox MA, Lewis KO, Cooper BT. Measurement of small intestinal permeability markers, lactulose, and mannitol in serum: results in celiac disease. Dig Dis Sci. 1999;44(2):402-406.

17. European Food Safety Authority. Opinion of the scientific panel on food additives, flavourings, processing aids and materials in contact with food on a request from the commission related to an application on the use of polyethylene glycol (PEG) as a film coating agent for use in food supplement products. Available at: http://www.efsa.europa.eu/ en/scdocs/doc/afc_op_ej414_polyethylene_glycol_sum_en\%20,0.pdf. Accessed June 5, 2012.

18. Castle L, Cloke HR, Crews C, Gilbert J. The migration of propylene glycol, mono-, di-, and triethylene glycols from regenerated cellulose film into food. Z Lebensm Unters Forsch. 1988;187(5):463-467. German.

19. Chadwick VS, Phillips SF, Hofmann AF. Measurements of intestinal permeability using low molecular weight polyethylene glycols (PEG 400). I. Chemical analysis and biological properties of PEG 400. Gastroenterology. 1977;73(2):241-246.

20. Chadwick VS, Phillips SF, Hofmann AF. Measurements of intestinal permeability using low molecular weight polyethylene glycols (PEG 400). II. Application to normal and abnormal permeability states in man and animals. Gastroenterology. 1977;73(2):247-251.

21. Bjarnason I, Williams P, Smethurst P, Peters TJ, Levi AJ. Effect of non-steroidal anti-inflammatory drugs and prostaglandins on the permeability of the human small intestine. Gut. 1986;27(11):1292-1297.

22. Bjarnason I, Takeuchi K. Intestinal permeability in the pathogenesis of NSAID-induced enteropathy. J Gastroenterol. 2009;44 Suppl 19: 23-29.

23. Parlesak A, Bode JC, Bode C. Parallel determination of gut permeability in man with $\mathrm{M}(\mathrm{r}) 400, \mathrm{M}(\mathrm{r}) 1500, \mathrm{M}(\mathrm{r}) 4000$ and $\mathrm{M}(\mathrm{r}) 10,000$ polyethylene glycol. Eur J Clin Chem Clin Biochem. 1994;32(11):813-820.

24. Kerckhoffs AP, Akkermans LM, de Smet MB, et al. Intestinal permeability in irritable bowel syndrome patients: effects of NSAIDs. Dig Dis Sci. 2010;55(3):716-723.

25. Pelsers MM, Hermens WT, Glatz JF. Fatty acid-binding proteins as plasma markers of tissue injury. Clin Chim Acta. 2005;352(1-2):15-35.

26. van Nieuwenhoven MA, de Swart EA, van Eijk HM, Deutz NE, Brouns F, Brummer RJ. Effects of pre- and post-absorptive factors on the lactulose/rhamnose gut permeability test. Clin Sci (Lond). 2000;98(3):349-353.

27. Garcia Rodriguez LA, Jick H. Risk of upper gastrointestinal bleeding and perforation associated with individual non-steroidal antiinflammatory drugs. Lancet. 1994;343(8900):769-772.

28. Loret S, Nollevaux G, Remacle R, et al. Analysis of PEG 400 and 4000 in urine for gut permeability assessment using solid phase extraction and gel permeation chromatography with refractometric detection. J Chromatogr B Analyt Technol Biomed Life Sci. 2004;805(2):195-202.

29. Lee S, Son SC, Han MJ, et al. Increased intestinal macromolecular permeability and urine nitrite excretion associated with liver cirrhosis with ascites. World J Gastroenterol. 2008;14(24):3884-3890.

30. Menzies IS, Zuckerman MJ, Nukajam WS, et al. Geography of intestinal permeability and absorption. Gut. 1999;44(4):483-489.

31. Ma TY, Hollander D, Krugliak P, Katz K. PEG 400, a hydrophilic molecular probe for measuring intestinal permeability. Gastroenterology. 1990;98(1):39-46.

32. Bijlsma PB, Fihn BM, Sjoqvist A, Groot JA, Taminiau JA, Jodal M. Water absorption enhances the uptake of mannitol and decreases Cr-EDTA/mannitol permeability ratios in cat small intestine in situ. Scand J Gastroenterol. 2002;37(7):799-806.

33. Bours MJ, Bos HJ, Meddings JB, Brummer RJ, van den Brandt PA, Dagnelie PC. Effects of oral adenosine 5 -triphosphate and adenosine in enteric-coated capsules on indomethacin-induced permeability changes in the human small intestine: a randomized cross-over study. BMC Gastroenterol. 2007;7:23.

34. Troost FJ, Saris WH, Brummer RJ. Recombinant human lactoferrin ingestion attenuates indomethacin-induced enteropathy in vivo in healthy volunteers. Eur J Clin Nutr. 2003;57(12):1579-1585. 
35. Playford RJ, MacDonald CE, Calnan DP, et al. Co-administration of the health food supplement, bovine colostrum, reduces the acute non-steroidal anti-inflammatory drug-induced increase in intestinal permeability. Clin Sci (Lond). 2001;100(6):627-633.

36. Marchbank T, Limdi JK, Mahmood A, Elia G, Playford RJ. Clinical trial: protective effect of a commercial fish protein hydrolysate against indomethacin (NSAID)-induced small intestinal injury. Aliment Pharmacol Ther. 2008;28(6):799-804
37. Mahmood A, Fitzgerald AJ, Marchbank T, et al. Zinc carnosine, a health food supplement that stabilises small bowel integrity and stimulates gut repair processes. Gut. 2007;56(2):168-175.

38. Bjarnason I, Maxton D, Reynolds AP, Catt S, Peters TJ, Menzies IS. Comparison of four markers of intestinal permeability in control subjects and patients with coeliac disease. Scand J Gastroenterol. 1994;29(7):630-639.

\section{Publish your work in this journal}

Clinical and Experimental Gastroenterology is an international, peerreviewed, open access journal, publishing all aspects of gastroenterology in the clinic and laboratory, including: Pathology, pathophysiology of gastrointestinal disease; Investigation and treatment of gastointestinal disease; Pharmacology of drugs used in the alimentary tract;
Immunology/genetics/genomics related to gastrointestinal disease. This journal is indexed on CAS. The manuscript management system is completely online and includes a very quick and fair peer-review system. Visit http://www.dovepress.com/testimonials.php to read rea quotes from published authors.

Submit your manuscript here: http://www.dovepress.com/clinical-and-experimental-gastroenterology-journal 